\title{
ON VOLUMES OF HYPERBOLIC COXETER POLYTOPES AND QUADRATIC FORMS
}

\author{
JOHN G. RATCLIFFE AND STEVEN T. TSCHANTZ
}

\begin{abstract}
In this paper, we compute the covolume of the group of units of the quadratic form $f_{d}^{n}(x)=x_{1}^{2}+x_{2}^{2}+\cdots+x_{n}^{2}-d x_{n+1}^{2}$ with $d$ an odd, positive, square-free integer. Mcleod has determined the hyperbolic Coxeter fundamental domain of the reflection subgroup of the group of units of the quadratic form $f_{3}^{n}$. We apply our covolume formula to compute the volumes of these hyperbolic Coxeter polytopes.
\end{abstract}

\section{INTRODUCTION}

In 1996, we computed the covolume of the group of units of the quadratic form

$$
f_{d}^{n}(x)=x_{1}^{2}+x_{2}^{2}+\cdots+x_{n}^{2}-d x_{n+1}^{2}
$$

with $d$ an odd, positive, square-free integer, in order to determine the volume of some hyperbolic Coxeter simplices, see [14] and $\S 5$ of [8]. We never got around to writing up our computation except for the case $d=1$ in [14. Recently, several papers have been written concerning the group of units of the quadratic form $f_{3}^{n}$, and so we felt it was time to write up our more general computation. Our main result is Theorem 4 which gives an explicit formula for the covolume of the group of units of $f_{d}^{n}$.

J. Mcleod [10] has determined the reflection subgroup of the group of units of $f_{3}^{n}$ and has shown that it has finite index if and only if $n \leq 13$. As an application of our computation, we determine the volume of the hyperbolic Coxeter fundamental domain of the reflection subgroup of the group of units of the quadratic form $f_{3}^{n}$, for $n \leq 13$, described in [10. See Table 1 for the volumes of Mcleod's polytopes.

M. Belolipetsky and V. Emery [2] proved that for each odd dimension $n \geq$ 5 , there is a unique, orientable, noncompact, arithmetic, hyperbolic $n$-orbifold of smallest volume and determined its volume. V. Emery mentioned in 6 that for $n \equiv 3 \bmod 4$, with $n \geq 7$, the corresponding arithmetic group is commensurable to the group of units of $f_{3}^{n}$. We determine the ratio between these two covolumes.

\section{Siegel's Covolume Formula for Unit Groups}

Let $f$ be a quadratic form in $n+1$ real variables, with $n \geq 2$, that is equivalent over $\mathbb{R}$ to the Lorentzian quadratic form

$$
x_{1}^{2}+\cdots+x_{n}^{2}-x_{n+1}^{2} .
$$

Let $S$ be the matrix of the quadratic form $f$. We assume that all the entries of $S$ are integers and $d=|\operatorname{det} S|$ is an odd, square-free, positive integer.

The group of units of the form $f$ is the group of all $(n+1) \times(n+1)$ matrices $A$, with integral entries, such that $A^{t} S A=S$. A unit of $f$ is said to be positive or negative according as $A$ leaves invariant each of the two connected components 
of $\left\{x \in \mathbb{R}^{n+1}: x^{t} S x<0\right\}$ or interchanges them. The group of positive units of $f$ corresponds to a discrete group $\Gamma$ of isometries of hyperbolic $n$-space

$$
H^{n}=\left\{x \in E^{n+1}: x_{1}^{2}+\cdots+x_{n}^{2}-x_{n+1}^{2}=-1 \text { and } x_{n+1}>0\right\}
$$

under the equivalence of $f$ with the Lorentzian quadratic form. For a discussion, see $\S 1$ of 9 ].

Let $q$ be a positive integer and let $\mathbb{Z}_{q}=\mathbb{Z} / q \mathbb{Z}$. Let $E_{q}(S)$ be the number of $(n+1) \times(n+1)$ matrices $A$ over $\mathbb{Z}_{q}$ such that $A^{t} S A=S$. By Formula 82 in Siegel's paper [16], we have that

$$
\operatorname{vol}\left(H^{n} / \Gamma\right)=4 d^{\frac{n+2}{2}} \prod_{k=1}^{n} \pi^{-\frac{k}{2}} \Gamma\left(\frac{k}{2}\right) \cdot \lim _{q \rightarrow \infty} 2^{\omega(q)} \frac{q^{\frac{n(n+1)}{2}}}{E_{q}(S)}
$$

where $\Gamma\left(\frac{k}{2}\right)$ is the gamma function evaluated at $k / 2$, and $\omega(q)$ is the number of distinct prime divisors of $q$, and $q$ goes to infinity via the sequence $2 !, 3 !, 4 ! \ldots$ In this paper a centered dot will delimit a product from the next factor.

We now evaluate the limit in Siegel's volume formula. Let $q=p_{1}^{a_{1}} p_{2}^{a_{2}} \cdots p_{r}^{a_{r}}$ be the prime factorization of $q$. Now $E_{q}(S)$ is a multiplicative function of $q$ by Lemma 18 of Siegel's paper [15], and so we have

$$
\frac{2^{\omega(q)} q^{\frac{n(n+1)}{2}}}{E_{q}(S)}=\prod_{i=1}^{r} \frac{2 p_{i}^{\frac{a_{i} n(n+1)}{2}}}{E_{p_{i}^{a_{i}}(S)}}
$$

Henceforth $p$ is a prime number. By Lemma 18 of [15], we have

$$
\frac{2 p^{\frac{a n(n+1)}{2}}}{E_{p^{a}}(S)}= \begin{cases}\frac{2 p^{\frac{3 n(n+1)}{2}}}{E_{p^{3}(S)}} & \text { if } p \mid 2 d \text { and } a \geq 3, \\ \frac{2 p^{\frac{n(n+1)}{2}}}{E_{p}(S)} & \text { if } p \nmid 2 d \text { and } a \geq 1 .\end{cases}
$$

Therefore, we have

$$
\lim _{q \rightarrow \infty} 2^{\omega(q)} \frac{q^{\frac{n(n+1)}{2}}}{E_{q}(S)}=\prod_{p \mid 2 d} \frac{2 p^{\frac{3 n(n+1)}{2}}}{E_{p^{3}}(S)} \prod_{p \nmid 2 d} \frac{2 p^{\frac{n(n+1)}{2}}}{E_{p}(S)} .
$$

First assume that $n$ is even and $p \nmid 2 d$. Then by Lemma 18 of [15], we have that

$$
\frac{2 p^{\frac{n(n+1)}{2}}}{E_{p}(S)}=\prod_{k=1}^{\frac{n}{2}}\left(1-p^{-2 k}\right)^{-1} \text {. }
$$

Hence we have

$$
\prod_{p \nmid 2 d} \frac{2 p^{\frac{n(n+1)}{2}}}{E_{p}(S)}=\prod_{k=1}^{\frac{n}{2}} \prod_{p \nmid 2 d}\left(1-p^{-2 k}\right)^{-1} .
$$

Using the product formula for the Riemann zeta function $\zeta(2 k)$, we have

$$
\prod_{p \nmid 2 d} \frac{2 p^{\frac{n(n+1)}{2}}}{E_{p}(S)}=\prod_{k=1}^{\frac{n}{2}}\left(\prod_{p \mid 2 d}\left(1-p^{-2 k}\right)\right) \zeta(2 k) .
$$


Now assume that $n$ is odd and $p \nmid 2 d$. Then by Lemma 18 of [15], we have that

$$
\frac{2 p^{\frac{n(n+1)}{2}}}{E_{p}(S)}=\left(1-\left(\frac{(-1)^{\frac{n-1}{2}} d}{p}\right) p^{-\left(\frac{n+1}{2}\right)}\right)^{-1} \prod_{k=1}^{\frac{n-1}{2}}\left(1-p^{-2 k}\right)^{-1}
$$

where $( \pm d / p)$ is a Legendre symbol. Define a fundamental discriminant $D$ by the formula

$$
D= \begin{cases}(-1)^{\frac{n-1}{2}} d & \text { if }(-1)^{\frac{n-1}{2}} d \equiv 1 \bmod 4 \\ 4(-1)^{\frac{n-1}{2}} d & \text { if }(-1)^{\frac{n-1}{2}} d \equiv 3 \bmod 4\end{cases}
$$

For all odd primes $p$, we have

$$
\left(\frac{(-1)^{\frac{n-1}{2}} d}{p}\right)=\left(\frac{D}{p}\right)
$$

Hence we have

$$
\prod_{p \nmid 2 d} \frac{2 p^{\frac{n(n+1)}{2}}}{E_{p}(S)}=\prod_{p \nmid 2 d}\left(1-\left(\frac{D}{p}\right) p^{-\left(\frac{n+1}{2}\right)}\right)^{-1} \prod_{k=1}^{\frac{n-1}{2}}\left(1-p^{-2 k}\right)^{-1} .
$$

Consider the Dirichlet $L$-series

$$
L(s, D)=\sum_{k=1}^{\infty}\left(\frac{D}{k}\right) k^{-s}
$$

where $(D / k)$ is a Kronecker symbol. This series converges absolutely for $s>1$. The Kronecker symbol $(D / k)$ is a completely multiplicative function of $k$ by Theorem 1.4.9 in Cohen [4. By Theorem 11.7 in Apostol [1, this $L$-function has the product formula

$$
L(s, D)=\prod_{p}\left(1-\left(\frac{D}{p}\right) p^{-s}\right)^{-1}
$$

Using the product formulas for $\zeta(2 k)$ and $L\left(\frac{n+1}{2}, D\right)$, we obtain the formula

$$
\prod_{p \nmid 2 d} \frac{2 p^{\frac{n(n+1)}{2}}}{E_{p}(S)}=\left(1-\left(\frac{D}{2}\right) 2^{-\left(\frac{n+1}{2}\right)}\right) L\left(\frac{n+1}{2}, D\right) \prod_{k=1}^{\frac{n-1}{2}}\left(\prod_{p \mid 2 d}\left(1-p^{-2 k}\right)\right) \zeta(2 k) .
$$

It remains only to compute $E_{p^{3}}(S)$ for each prime number $p$ dividing $2 d$.

\section{The computation of $E_{8}(S)$}

From now on, we assume that $f=f_{d}^{n}$. Then $S$ is the $(n+1) \times(n+1)$ diagonal matrix $\operatorname{diag}(1, \ldots, 1,-d)$. Let $k$ be a positive integer, and let $\mathbb{Z}_{k}=\mathbb{Z} / k \mathbb{Z}$. Let $\mathrm{O}\left(n+1, \mathbb{Z}_{k}\right)$ be the group of $(n+1) \times(n+1)$ matrices $A$ over the ring $\mathbb{Z}_{k}$ such that $A^{t} A=I$. Let $J$ be the $(n+1) \times(n+1)$ diagonal matrix $\operatorname{diag}(1, \ldots, 1,-1)$. Let $\mathrm{O}\left(n, 1 ; \mathbb{Z}_{k}\right)$ be the group of $(n+1) \times(n+1)$ matrices $A$ over the ring $\mathbb{Z}_{k}$ such that $A^{t} J A=J$.

Let $\mathrm{O}\left(S, \mathbb{Z}_{k}\right)$ be the group of invertible $(n+1) \times(n+1)$ matrices $A$ over the ring $\mathbb{Z}_{k}$ such that $A^{t} S A=S$. Then $E_{8}(S)$ is the order of $\mathrm{O}\left(S, \mathbb{Z}_{8}\right)$, since $d$ is odd.

Let $\mathrm{O}^{*}\left(n+1, \mathbb{Z}_{2}\right)$ be the image of $\mathrm{O}\left(n+1, \mathbb{Z}_{4}\right)$ in $\mathrm{O}\left(n+1, \mathbb{Z}_{2}\right)$ under the homomorphism induced by the ring homomorphism from $\mathbb{Z}_{4}$ to $\mathbb{Z}_{2}$, and let $\mathrm{O}^{*}\left(n, 1 ; \mathbb{Z}_{2}\right)$ 
be the image of $\mathrm{O}\left(n, 1 ; \mathbb{Z}_{4}\right)$ in $\mathrm{O}\left(n+1, \mathbb{Z}_{2}\right)$ under the homomorphism induced by the ring homomorphism from $\mathbb{Z}_{4}$ to $\mathbb{Z}_{2}$.

We know that reduction modulo 2 maps $\mathrm{O}\left(S, \mathbb{Z}_{8}\right)$ into $\mathrm{O}^{*}\left(n, 1 ; \mathbb{Z}_{2}\right)$ if $d \equiv 1 \bmod 4$ and into $\mathrm{O}^{*}\left(n+1, \mathbb{Z}_{2}\right)$ if $d \equiv-1 \bmod 4$, since the image of $\mathrm{O}\left(S, \mathbb{Z}_{8}\right)$ factors through $\mathrm{O}\left(S, \mathbb{Z}_{4}\right)$. We next show that $\mathrm{O}\left(S, \mathbb{Z}_{8}\right)$ maps onto the appropriate $\mathrm{O}^{*}$ subgroup of $\mathrm{O}\left(n+1, \mathbb{Z}_{2}\right)$ under reduction modulo 2 .

Lemma 1. If $d \equiv-1 \bmod 4$, then $\mathrm{O}\left(S, \mathbb{Z}_{8}\right)$ maps onto $\mathrm{O}^{*}\left(n+1, \mathbb{Z}_{2}\right)$ under reduction modulo 2.

Proof. By the discussion on page 60 of [14], the group $\mathrm{O}^{*}\left(n+1, \mathbb{Z}_{2}\right)$ is generated by the permutation matrices and, if $n+1 \geq 6$, a matrix $C$ which is the identity except for a lower corner $6 \times 6$ block with all 0 diagonal and all 1 off-diagonal entries.

First assume that $d \equiv-1 \bmod 8$. Then $\mathrm{O}\left(S, \mathbb{Z}_{8}\right)=\mathrm{O}\left(n+1, \mathbb{Z}_{8}\right)$. The permutation matrices in $\mathrm{O}^{*}\left(n+1, \mathbb{Z}_{2}\right)$ obviously lift to $\mathrm{O}\left(n+1, \mathbb{Z}_{8}\right)$. If $n+1 \geq 6$, the matrix $C$ lifts to $\mathrm{O}\left(n+1, \mathbb{Z}_{8}\right)$, since the identity part of $C$ lifts as is, and the $6 \times 6$ block lifts to a block with all 2 diagonal and all 1 off-diagonal entries.

Now assume that $d \equiv 3 \bmod 8$. First we argue that all the permutation matrices lift to $\mathrm{O}\left(S, \mathbb{Z}_{8}\right)$. Clearly the permutations of the first $n$ coordinates lift. Thus it suffices to show that the transposition of the last two coordinates also lifts, and the lift is the $2 \times 2$ block with diagonal 2, 6 and all 1 off-diagonal entries, that is,

$$
\left(\begin{array}{ll}
2 & 1 \\
1 & 6
\end{array}\right)\left(\begin{array}{rr}
1 & 0 \\
0 & -3
\end{array}\right)\left(\begin{array}{rr}
2 & 1 \\
1 & 6
\end{array}\right) \equiv\left(\begin{array}{rr}
1 & 0 \\
0 & -3
\end{array}\right) \bmod 8
$$

We also need our lower $6 \times 6$ corner block to lift. The lift has diagonal entries $0,0,0,0,0,4$ and all 1 off-diagonal entries.

Lemma 2. If $d \equiv 1 \bmod 4$, then $\mathrm{O}\left(S, \mathbb{Z}_{8}\right)$ maps onto $\mathrm{O}^{*}\left(n, 1 ; \mathbb{Z}_{2}\right)$ under reduction modulo 2.

Proof. The proof for the case $d \equiv 1 \bmod 8$ follows from Lemma 4 in [14. Now assume $d \equiv-3 \bmod 8$. Identify $\mathrm{O}^{*}\left(n, \mathbb{Z}_{2}\right)$ with the subgroup of $\mathrm{O}^{*}\left(n, 1 ; \mathbb{Z}_{2}\right)$ fixing the last standard basis vector $e_{n+1}$. By the proof of Lemma 4 in [14, the group $\mathrm{O}^{*}\left(n, 1 ; \mathbb{Z}_{2}\right)$ is generated by the subgroup $\mathrm{O}^{*}\left(n, \mathbb{Z}_{2}\right)$ and a matrix $C$ which is the identity except for a lower corner $4 \times 4$ block with all 0 diagonal and all 1 off-diagonal entries. The subgroup $\mathrm{O}^{*}\left(n, \mathbb{Z}_{2}\right)$ lifts to $\mathrm{O}\left(S, \mathbb{Z}_{8}\right)$ as in the case $d \equiv-1 \bmod 8$ in Lemma 1 . The $4 \times 4$ lower corner block of $C$ lifts to the $4 \times 4$ block with diagonal 2, 2, 2, 4 and all 1 off-diagonal entries.

Lemma 3. Let $\eta: \mathrm{O}\left(S, \mathbb{Z}_{8}\right) \rightarrow \mathrm{O}\left(S, \mathbb{Z}_{2}\right)$ be the homomorphism induced by reduction modulo 2. The kernel of $\eta$ has order $2^{n^{2}+2 n+2}$.

Proof. Each matrix $M$ in $\mathrm{O}\left(S, \mathbb{Z}_{8}\right)$ can be written uniquely in the form $N+2 U+4 V$ where $N, U$, and $V$ are zero-one matrices and we are interested in counting the $M$ with $N=I$. Now observe that

$$
M^{t} S M=S+2\left(U^{t} S+S U\right)+4\left(U^{t} S U+V^{t} S+S V\right) .
$$

Using the fact that $4(-d) \equiv 4 \bmod 8$, the last equation simplifies to

$$
M^{t} S M=S+2\left(U^{t} S+S U\right)+4\left(U^{t} U+V^{t}+V\right) .
$$

First assume that $d \equiv 1 \bmod 4$. Then $2(-d) \equiv 2(-1) \bmod 8$ and the last equation simplifies to

$$
M^{t} S M=S+2\left(U^{t} J+J U\right)+4\left(U^{t} U+V^{t}+V\right) .
$$


The proof now proceeds as in the proof of Theorem 5 of [14].

Now assume that $d \equiv-1 \bmod 4$. Then $2(-d) \equiv 2 \bmod 8$ and the next to last equation simplifies to

$$
M^{t} S M=S+2\left(U^{t}+U\right)+4\left(U^{t} U+V^{t}+V\right) .
$$

The proof now proceeds as in the proof of Theorem 5 of [14] with the simplification that $W=0$.

Let $\epsilon_{2}(k)=1$ if $k$ is even and 0 if $k$ is odd and define

$$
\alpha(n)=\prod_{k=1}^{n}\left(2^{k}-\epsilon_{2}(k)\right) \text {. }
$$

Define

$$
\beta(n)=2^{n-2}+2^{(n-2) / 2} \cos (n \pi / 4),
$$

and

$$
\gamma(n)=2^{n}+2^{n / 2} \cos (n \pi / 4) .
$$

Note that the function $f(n)=\cos (n \pi / 4)$ is periodic with period 8 . The values of $f(n)$ for $n=0,1, \ldots, 7$ are $1,2^{-1 / 2}, 0,-2^{-1 / 2},-1,-2^{-1 / 2}, 0,2^{-1 / 2}$, respectively.

Theorem 1. The value of $E_{8}(S)$ is given by

$$
E_{8}(S)= \begin{cases}2^{n^{2}+2 n+2} \alpha(n) / \beta(n+1) & \text { if } d \equiv-1 \bmod 4, \\ 2^{n^{2}+2 n+2} \alpha(n) / \gamma(n-1) & \text { if } d \equiv 1 \bmod 4 .\end{cases}
$$

Proof. By Lemmas 2 of [14], the order of $\mathrm{O}^{*}\left(n+1, \mathbb{Z}_{2}\right)$ is $\alpha(n) / \beta(n+1)$, and by Lemma 3 of [14, the order $\mathrm{O}^{*}\left(n, 1 ; \mathbb{Z}_{2}\right)$ is $\alpha(n) / \gamma(n-1)$. The Theorem now follows from Lemmas 1, 2, 3 .

Corollary 1. We have that

$$
\frac{2 \cdot 2^{\frac{3 n(n+1)}{2}}}{E_{8}(S)}=\frac{2^{\frac{n-1}{2}}+\cos \left(\left(n+(-1)^{\frac{d+1}{2}}\right) \pi / 4\right)}{2^{\frac{n+3}{2}} \prod_{k=1}^{\left[\frac{n}{2}\right]}\left(1-2^{-2 k}\right)} .
$$

Proof. By Theorem 1, we have that

$$
\frac{2 \cdot 2^{\frac{3 n(n+1)}{2}}}{E_{8}(S)}=\frac{2 \cdot 2^{\frac{3 n(n+1)}{2}}\left(2^{n-1}+2^{\frac{n-1}{2}} \cos \left(\left(n+(-1)^{\frac{d+1}{2}}\right) \pi / 4\right)\right)}{2^{n^{2}+2 n+2} \prod_{k=1}^{n}\left(2^{k}-\epsilon_{2}(k)\right)} .
$$

If $n$ is odd, we have that

$$
\begin{aligned}
\prod_{k=1}^{n}\left(2^{k}-\epsilon_{2}(k)\right) & =2 \prod_{k=1}^{\frac{n-1}{2}} 2^{2 k+1}\left(2^{2 k}-1\right) \\
& =2 \prod_{k=1}^{\frac{n-1}{2}} 2^{4 k+1}\left(1-2^{-2 k}\right)=2^{\frac{n(n+1)}{2}} \prod_{k=1}^{\frac{n-1}{2}}\left(1-2^{-2 k}\right) .
\end{aligned}
$$


If $n$ is even, we have that

$$
\begin{aligned}
\prod_{k=1}^{n}\left(2^{k}-\epsilon_{2}(k)\right) & =\prod_{k=1}^{\frac{n}{2}} 2^{2 k-1}\left(2^{2 k}-1\right) \\
& =\prod_{k=1}^{\frac{n}{2}} 2^{4 k-1}\left(1-2^{-2 k}\right)=2^{\frac{n(n+1)}{2}} \prod_{k=1}^{\frac{n}{2}}\left(1-2^{-2 k}\right) .
\end{aligned}
$$

Therefore, we have that

$$
\begin{aligned}
\frac{2 \cdot 2^{\frac{3 n(n+1)}{2}}}{E_{8}(S)}= & \frac{2 \cdot 2^{\frac{3 n(n+1)}{2}} 2^{\frac{n-1}{2}}\left(2^{\frac{n-1}{2}}+\cos \left(\left(n+(-1)^{\frac{d+1}{2}}\right) \pi / 4\right)\right)}{2^{n^{2}+2 n+2} 2^{\frac{n(n+1)}{2}} \prod_{k=1}^{\left[\frac{n}{2}\right]}\left(1-2^{-2 k}\right)} \\
= & \frac{2^{\frac{n-1}{2}}+\cos \left(\left(n+(-1)^{\frac{d+1}{2}}\right) \pi / 4\right)}{2^{\frac{n+3}{2}} \prod_{k=1}^{\left[\frac{n}{2}\right]}\left(1-2^{-2 k}\right)}
\end{aligned}
$$

4. The computation of $E_{p^{3}}(S)$

Let $p$ be a prime number that divides the odd, positive, square-free integer $d$. In this section, we determine the value of $E_{p^{3}}(S)$.

Let $a$ be a positive integer with $a \geq 2$, and let $M \in \mathrm{GL}\left(n+1, \mathbb{Z}_{p^{a}}\right)$. Define

$$
\begin{aligned}
& \alpha(M)=\operatorname{diag}(1,1, \ldots, 1,0) \cdot M, \\
& \beta(M)=\operatorname{diag}(0,0, \ldots, 0,1) \cdot M .
\end{aligned}
$$

Then $\alpha(M)$ is the matrix $M$ with its last row set to zero, and $\beta(M)$ is $M$ with its first $n$ rows set to zero. Note that $M=\alpha(M)+\beta(M)$.

Define $\bar{\beta}(M)=\beta(M)$ reduced modulo $p^{a-1}$ with entries in the range $\left[0, p^{a-1}\right)$. Define $\bar{M}=\alpha(M)+\bar{\beta}(M)$. Then $M-\bar{M}=p^{a-1} U$ for $U=\beta(U)$ with entries in the range $[0, p)$. Define

$$
\overline{\mathrm{O}}\left(S, \mathbb{Z}_{p^{a}}\right)=\left\{\bar{M}: M \in \mathrm{O}\left(S, \mathbb{Z}_{p^{a}}\right)\right\} .
$$

Lemma 4. Let $M \in \mathrm{GL}\left(n+1, \mathbb{Z}_{p^{a}}\right)$ with $a \geq 2$. Then $M \in \mathrm{O}\left(S, \mathbb{Z}_{p^{a}}\right)$ if and only if $\bar{M} \in \mathrm{O}\left(S, \mathbb{Z}_{p^{a}}\right)$. Moreover

$$
E_{p^{a}}(S)=\left|\mathrm{O}\left(S, \mathbb{Z}_{p^{a}}\right)\right|=p^{n+1}\left|\overline{\mathrm{O}}\left(S, \mathbb{Z}_{p^{a}}\right)\right| .
$$

Proof. Let $M$ be an $(n+1) \times(n+1)$ matrix over $\mathbb{Z}$ such that $M^{t} S M \equiv S \bmod$ $p^{a}$. Then $(\operatorname{det}(M))^{2}(-d) \equiv-d \bmod p^{a}$. Hence $(\operatorname{det}(M))^{2} \equiv 1 \bmod p^{a-1}$, and so $\operatorname{det}(M)$ is invertible modulo $p^{a}$. Hence the reduction of $M$ modulo $p^{a}$ is invertible. Therefore $E_{p^{a}}(S)=\left|\mathrm{O}\left(S, \mathbb{Z}_{p^{a}}\right)\right|$.

Now suppose $M$ is an $(n+1) \times(n+1)$ matrix over $\mathbb{Z}_{p^{a}}$. Observe that

$$
\begin{aligned}
M^{t} S M & =\left(\bar{M}+p^{a-1} U\right)^{t} S\left(\bar{M}+p^{a-1} U\right) \\
& =\bar{M}^{t} S \bar{M}+p^{a-1} U^{t} S \bar{M}+p^{a-1} \bar{M}^{t} S U+p^{2 a-2} U^{t} S U \\
& =\bar{M}^{t} S \bar{M}+p^{a-1}(-d) U^{t} \bar{M}+p^{a-1}(-d) \bar{M}^{t} U \\
& =\bar{M}^{t} S \bar{M} .
\end{aligned}
$$


Thus $M \in \mathrm{O}\left(S, \mathbb{Z}_{p^{a}}\right)$ if and only if $\bar{M} \in \mathrm{O}\left(S, \mathbb{Z}_{p^{a}}\right)$. In the equation $M-\bar{M}=p^{a-1} U$, there are $p^{n+1}$ choices for $U$. Hence for each $\bar{M}$ there are $p^{n+1}$ choices for $M$. Therefore $\left|\mathrm{O}\left(S, \mathbb{Z}_{p^{a}}\right)\right|=p^{n+1}\left|\overline{\mathrm{O}}\left(S, \mathbb{Z}_{p^{a}}\right)\right|$.

Lemma 5. If $a \geq 2$, we have that $\left|\overline{\mathrm{O}}\left(S, \mathbb{Z}_{p^{a+1}}\right)\right|=p^{\frac{n(n+1)}{2}}\left|\overline{\mathrm{O}}\left(S, \mathbb{Z}_{p^{a}}\right)\right|$.

Proof. If $M \in \overline{\mathrm{O}}\left(S, \mathbb{Z}_{p^{a+1}}\right)$, then $N=\overline{\left(M \bmod p^{a}\right)} \in \overline{\mathrm{O}}\left(S, \mathbb{Z}_{p^{a}}\right)$. We want to count

$$
M=N+p^{a} \alpha(U)+p^{a-1} \beta(U)
$$

for $U \bmod p$ and $N \in \overline{\mathrm{O}}\left(S, \mathbb{Z}_{p^{a}}\right)$ such that $M \in \overline{\mathrm{O}}\left(S, \mathbb{Z}_{p^{a+1}}\right)$. Observe that

$$
\begin{aligned}
M^{t} S M-S & =N^{t} S N-S \\
& +p^{a}\left(\alpha(U)^{t} S N+N^{t} S \alpha(U)\right) \\
& +p^{a-1}\left(\beta(U)^{t} S N+N^{t} S \beta(U)\right) \\
& +p^{2 a-1}\left(\alpha(U)^{t} S \beta(U)+\beta(U)^{t} S \alpha(U)\right) \\
& +p^{2 a}\left(\alpha(U)^{t} S \alpha(U)\right)+p^{2 a-2}\left(\beta(U)^{t} S \beta(U)\right) \\
& =N^{t} S N-S \\
& +p^{a}\left(\alpha(U)^{t} N+N^{t} \alpha(U)\right) \\
& +p^{a}(-d / p)\left(\beta(U)^{t} N+N^{t} \beta(U)\right) .
\end{aligned}
$$

Let $V=\alpha(U)+(-d / p) \beta(U)$. Then $\alpha(U)=\alpha(V)$ and $\beta(U) \equiv(-d / p)^{-1} \beta(V) \bmod p$.

Now $M^{t} S M=S$ if and only if

$$
V^{t} N+N^{t} V \equiv \frac{S-N^{t} S N}{p^{a}} \bmod p
$$

where we regard the entries of $N$ to be integers in the range $\left[0, p^{a}\right)$. Let $W=N^{t} V$ $\bmod p$. Then $V=\left(N^{t}\right)^{-1} W \bmod p$. Note that $N^{t}$ is invertible mod $p$, since $N$ is invertible $\bmod p$ by Lemma 4 . Now $M^{t} S M=S$ if and only if

$$
W^{t}+W \equiv \frac{S-N^{t} S N}{p^{a}} \bmod p .
$$

This congruence determines the diagonal of $W$ and below diagonal entries in terms of arbitrary above diagonal entries for $p^{n(n+1) / 2}$ choices for $W$ with entries mod $p$. Thus we have $p^{n(n+1) / 2}$ choices for $U$ and as many $M \in \overline{\mathrm{O}}\left(S, \mathbb{Z}_{p^{a+1}}\right)$ for each $N \in \overline{\mathrm{O}}\left(S, \mathbb{Z}_{p^{a}}\right)$.

Lemma 6. We have that $\left|\overline{\mathrm{O}}\left(S, \mathbb{Z}_{p^{2}}\right)\right|=2 p^{\frac{n(n+1)}{2}}\left|\mathrm{O}\left(n, \mathbb{Z}_{p}\right)\right|$.

Proof. Let $M \in \overline{\mathrm{O}}\left(S, \mathbb{Z}_{p^{2}}\right)$ and decompose $M$ into blocks

$$
M=\left(\begin{array}{l|l}
M_{0} & v \\
\hline w^{t} & x
\end{array}\right)
$$

with $v \in \mathbb{Z}_{p^{2}}^{n}, w \in \mathbb{Z}_{p}^{n}$ and $x \in \mathbb{Z}_{p}$.

Observe that $M^{t} S M=S$ if and only if

$$
\begin{cases}M_{0}^{t} M_{0}-d w w^{t} & \equiv I \bmod p^{2} \\ M_{0}^{t} v-d x w & \equiv 0 \bmod p^{2} \\ v^{t} v-d x^{2} & \equiv-d \bmod p^{2}\end{cases}
$$


The above system of congruences implies that

$$
\begin{cases}M_{0}^{t} M_{0} & \equiv I \bmod p \\ v & \equiv 0 \bmod p \\ x^{2} & \equiv 1 \bmod p\end{cases}
$$

Let $N_{0}=\left(M_{0} \bmod p\right)$. Then $M_{0}=N_{0}+p U_{0}$ for $U_{0} \bmod p$ and $N_{0} \in \mathrm{O}\left(n, \mathbb{Z}_{p}\right)$. For each such $N_{0}$ choose $x= \pm 1 \bmod p$ and $w \in \mathbb{Z}_{p}^{n}$ arbitrarily. We need to count the $U_{0}$ and find $v$ so that $M \in \overline{\mathrm{O}}\left(S, \mathbb{Z}_{p^{2}}\right)$. Note that $v=p y$ where $M_{0}^{t} y \equiv(d / p) x w \bmod p$, and so $v$ is determined from $x, w$ and $N_{0}$. Thus it suffices to count $U_{0}$ such that

$$
\left(N_{0}+p U_{0}\right)^{t}\left(N_{0}+p^{b} U_{0}\right)-d w w^{t} \equiv I \bmod p^{2} .
$$

The above congruence is equivalent to

$$
U_{0}^{t} N_{0}+N_{0}^{t} U_{0} \equiv \frac{I-N_{0}^{t} N_{0}}{p}+(d / p) w w^{t} \bmod p .
$$

Let $V_{0}=N_{0}^{t} U_{0}$. Then $U_{0}=\left(N_{0}^{t}\right)^{-1} V_{0}$. The congruence

$$
V_{0}^{t}+V_{0} \equiv \frac{I-N_{0}^{t} N_{0}}{p}+(d / p) w w^{t} \bmod p
$$

determines the diagonal of $V_{0}$ and below diagonal entries from the above diagonal entries. Hence there are $p^{n(n-1) / 2}$ choices for $V_{0}$ and so for $U_{0}$ given $w$ and $x$. Thus there are $2 p^{n(n-1) / 2+n}$ possibilities for $M \in \overline{\mathrm{O}}\left(S, \mathbb{Z}_{p^{2}}\right)$ for each $N_{0} \in \mathrm{O}\left(n, \mathbb{Z}_{p}\right)$.

Theorem 2. The values of $E_{p^{2}}(S)$ and $E_{p^{3}}(S)$ are given by

$$
\begin{aligned}
& E_{p^{2}}(S)=2 p^{\frac{(n+1)(n+2)}{2}}\left|\mathrm{O}\left(n, \mathbb{Z}_{p}\right)\right|, \\
& E_{p^{3}}(S)=p^{\frac{n(n+1)}{2}} E_{p^{2}}(S) .
\end{aligned}
$$

Proof. By Lemmas 4 and 6, we have that

$$
\begin{aligned}
E_{p^{2}}(S) & =p^{n+1}\left|\overline{\mathrm{O}}\left(S, \mathbb{Z}_{p^{2}}\right)\right| \\
& =p^{n+1} 2 p^{\frac{n(n+1)}{2}}\left|\mathrm{O}\left(n, \mathbb{Z}_{p}\right)\right|=2 p^{\frac{(n+1)(n+2)}{2}}\left|\mathrm{O}\left(n, \mathbb{Z}_{p}\right)\right|
\end{aligned}
$$

By Lemmas 4 and 5, we have that

$$
\begin{aligned}
E_{p^{3}}(S) & =p^{n+1}\left|\overline{\mathrm{O}}\left(S, \mathbb{Z}_{p^{3}}\right)\right| \\
& =p^{n+1} p^{\frac{n(n+1)}{2}}\left|\mathrm{O}\left(S, \mathbb{Z}_{p^{2}}\right)\right|=p^{\frac{n(n+1)}{2}} E_{p^{2}}(S) .
\end{aligned}
$$

Corollary 2. We have that

$$
\frac{2 p^{\frac{3 n(n+1)}{2}}}{E_{p^{3}}(S)}=\frac{2 p^{\frac{2 n(n+1)}{2}}}{E_{p^{2}}(S)}=\frac{p^{\frac{(n+1)(n-2)}{2}}}{\left|\mathrm{O}\left(n, \mathbb{Z}_{p}\right)\right|} .
$$

We need the following classical theorem, see Theorem 172 of Dickson [5].

Theorem 3. If $p$ be an odd prime number, then the order of $\mathrm{O}\left(n, \mathbb{Z}_{p}\right)$ is given by

$$
\left|\mathrm{O}\left(n, \mathbb{Z}_{p}\right)\right|= \begin{cases}2 \prod_{k=1}^{n-1} p^{k}-\epsilon_{2}(k) & n \text { odd, } \\ 2 p^{\frac{n}{2}-1}\left(p^{\frac{n}{2}}-\left(\frac{-1}{p}\right)^{\frac{n}{2}}\right) \prod_{k=1}^{n-2} p^{k}-\epsilon_{2}(k) & n \text { even, }\end{cases}
$$

where $(-1 / p)$ is a Legendre symbol. 
Corollary 3. We have that

$$
\frac{2 p^{\frac{3 n(n+1)}{2}}}{E_{p^{3}}(S)}=\frac{p^{\frac{n}{2}}+\epsilon_{2}(n)\left(\frac{-1}{p}\right)^{\frac{n}{2}}}{2 p^{\frac{n+2}{2}} \prod_{k=1}^{\left[\frac{n}{2}\right]}\left(1-p^{-2 k}\right)} .
$$

Proof. First assume that $n$ is odd. By Theorem 3 and Corollary 2, we have that

$$
\begin{aligned}
\frac{2 p^{\frac{3 n(n+1)}{2}}}{E_{p^{3}}(S)} & =\frac{p^{\frac{(n+1)(n-2)}{2}}}{2 \prod_{k=1}^{n-1} p^{k}-\epsilon_{2}(k)} \\
& =\frac{p^{\frac{(n+1)(n-2)}{2}}}{2 \prod_{k=1}^{\frac{n-1}{2}} p^{2 k-1}\left(p^{2 k}-1\right)} \\
& =\frac{p^{\frac{(n+1)(n-2)}{2}}}{\prod_{k=1}^{\frac{n-1}{2}} p^{4 k-1}\left(1-p^{-2 k}\right)} \\
& =\frac{p^{\frac{(n+1)(n-2)}{2}}}{2 p^{\frac{(n-1) n}{2}} \prod_{k=1}^{\frac{n-1}{2}}\left(1-p^{-2 k}\right)}=\frac{2 p \prod_{k=1}^{\frac{n-1}{2}}\left(1-p^{-2 k}\right)}{} .
\end{aligned}
$$

Now assume that $n$ is even. By Theorem 3 and Corollary 2, we have that

$$
\begin{aligned}
\frac{2 p^{\frac{3 n(n+1)}{2}}}{E_{p^{3}(S)}} & =\frac{p^{\frac{(n+1)(n-2)}{2}}}{2 p^{\frac{n}{2}-1}\left(p^{\frac{n}{2}}-\left(\frac{-1}{p}\right)^{\frac{n}{2}}\right) \prod_{k=1}^{n-2} p^{k}-\epsilon_{2}(k)} \\
& =\frac{p^{\frac{(n+1)(n-2)}{2}\left(p^{\frac{n}{2}}+\left(\frac{-1}{p}\right)^{\frac{n}{2}}\right)}}{2 p^{\frac{n}{2}-1}\left(p^{n}-1\right) \prod_{k=1}^{n-2} p^{k}-\epsilon_{2}(k)} \\
& =\frac{p^{\frac{(n+1)(n-2)}{2}}\left(p^{\frac{n}{2}}+\left(\frac{-1}{p}\right)^{\frac{n}{2}}\right)}{2 p^{-\frac{n}{2}} \prod_{k=1}^{n} p^{k}-\epsilon_{2}(k)} \\
& =\frac{p^{\frac{(n+1)(n-2)}{2}}\left(p^{\frac{n}{2}}+\left(\frac{-1}{p}\right)^{\frac{n}{2}}\right)}{2 p^{-\frac{n}{2}} p^{\frac{n(n+1)}{2}} \prod_{k=1}^{\frac{n}{2}}\left(1-p^{-2 k}\right)}=\frac{p^{\frac{n}{2}}+\left(\frac{-1}{p}\right)^{\frac{n}{2}}}{2 p^{\frac{n+2}{2}} \prod_{k=1}^{\frac{n}{2}}\left(1-p^{-2 k}\right)} .
\end{aligned}
$$




\section{The covolume of the group of units of $f_{d}^{n}$}

Let $\Gamma_{d}^{n}$ be the discrete group of isometries of hyperbolic $n$-space $H^{n}$ that corresponds to the group of positive units of the quadratic form $f_{d}^{n}$. In this section, we give an explicit formula for $\operatorname{vol}\left(H^{n} / \Gamma_{d}^{n}\right)$.

From Formula (6) of [14], we have

$$
\prod_{k=1}^{n} \pi^{-\frac{k}{2}} \Gamma\left(\frac{k}{2}\right)= \begin{cases}\prod_{k=1}^{\frac{n-1}{2}} \frac{2(2 k-1) !}{(2 \pi)^{2 k}} & n \text { odd } \\ \prod_{k=1}^{\frac{n}{2}} \frac{2(2 k-1) !}{(2 \pi)^{2 k}} \cdot \frac{(2 \pi)^{\frac{n}{2}}}{(n-1) ! !} & n \text { even }\end{cases}
$$

By Theorems 12.17 and 12.18 of [1], we have that

$$
\zeta(2 k)=\frac{(2 \pi)^{2 k}\left|B_{2 k}\right|}{2(2 k) !}
$$

for every positive integer $k$ where $B_{2 k}$ is the $(2 k)$ th Bernoulli number.

Define a function $B$ of $n$ by the formula

$$
B=\prod_{k=1}^{\left[\frac{n}{2}\right]} \frac{\left|B_{2 k}\right|}{2 k}
$$

Then we have that

$$
\prod_{k=1}^{n} \pi^{-\frac{k}{2}} \Gamma\left(\frac{k}{2}\right) \prod_{k=1}^{\left[\frac{n}{2}\right]} \zeta(2 k)= \begin{cases}B & n \text { odd } \\ B \cdot \frac{(2 \pi)^{\frac{n}{2}}}{(n-1) ! !} & n \text { even }\end{cases}
$$

Define a function $C$ of $n$ and $d$ by the formula

$$
C=\cos \left(\left(n+(-1)^{\frac{d+1}{2}}\right) \pi / 4\right) .
$$

Theorem 4. Let d be an odd, square-free, positive integer, and let $\Gamma_{d}^{n}$ be the discrete group of isometries of hyperbolic n-space $H^{n}$ corresponding to the group of positive units of the quadratic form $f_{d}^{n}$. The volume of $H^{n} / \Gamma_{d}^{n}$ is given by

$$
\operatorname{vol}\left(H^{n} / \Gamma_{d}^{n}\right)= \begin{cases}\frac{d^{\frac{n-1}{2}} B}{2^{n+\omega(d)}}\left(2^{\frac{n-1}{2}}+C\right)\left(2^{\frac{n+1}{2}}-\left(\frac{D}{2}\right)\right) \sqrt{d} \cdot L\left(\frac{n+1}{2}, D\right) & n \text { odd } \\ \frac{B}{2^{\frac{n}{2}+\omega(d)}}\left(2^{\frac{n}{2}}+2^{\frac{1}{2}} C\right) \prod_{p \mid d}\left(p^{\frac{n}{2}}+\left(\frac{-1}{p}\right)^{\frac{n}{2}}\right) \cdot \frac{(2 \pi)^{\frac{n}{2}}}{(n-1) ! !} & n \text { even } .\end{cases}
$$


Proof. First assume that $n$ is odd. From Formulas (1), (4), (14), (24) and Corollaries 1 and 3, we have that

$$
\begin{aligned}
\operatorname{vol}\left(H^{n} / \Gamma_{d}^{n}\right) & =4 d^{\frac{n+2}{2}} \prod_{k=1}^{n} \pi^{-\frac{k}{2}} \Gamma\left(\frac{k}{2}\right) \cdot \lim _{q \rightarrow \infty} 2^{\omega(q)} \frac{q^{\frac{n(n+1)}{2}}}{E_{q}(S)} \\
& =4 d^{\frac{n+2}{2}} \prod_{k=1}^{n} \pi^{-\frac{k}{2}} \Gamma\left(\frac{k}{2}\right) \cdot \prod_{p \mid 2 d} \frac{2 p^{\frac{3 n(n+1)}{2}}}{E_{p^{3}}(S)} \cdot \prod_{p \nmid 2 d} \frac{2 p^{\frac{n(n+1)}{2}}}{E_{p}(S)} \\
& =4 d^{\frac{n+2}{2}} B \prod_{p \mid 2 d} \frac{2 p^{\frac{3 n(n+1)}{2}}}{E_{p^{3}}(S)}\left(1-\left(\frac{D}{2}\right) 2^{-\left(\frac{n+1}{2}\right)}\right) L\left(\frac{n+1}{2}, D\right) \prod_{k=1}^{\frac{n-1}{2}} \prod_{p \mid 2 d}\left(1-p^{-2 k}\right) \\
& =4 d^{\frac{n+2}{2}} B \frac{\left(2^{\frac{n-1}{2}}+C\right)}{2^{\frac{n+3}{2}} 2^{\omega(d)} d}\left(1-\left(\frac{D}{2}\right) 2^{-\left(\frac{n+1}{2}\right)}\right) L\left(\frac{n+1}{2}, D\right) \\
& =\frac{d^{\frac{n-1}{2}} B}{2^{n+\omega(d)}}\left(2^{\frac{n-1}{2}}+C\right)\left(2^{\frac{n+1}{2}}-\left(\frac{D}{2}\right)\right) \sqrt{d} \cdot L\left(\frac{n+1}{2}, D\right) .
\end{aligned}
$$

Now assume that $n$ is even. From Formulas (1), (4), (7), (24) and Corollaries 1 and 3 , we have that

$$
\begin{aligned}
\operatorname{vol}\left(H^{n} / \Gamma_{d}^{n}\right) & =4 d^{\frac{n+2}{2}} \prod_{k=1}^{n} \pi^{-\frac{k}{2}} \Gamma\left(\frac{k}{2}\right) \cdot \prod_{p \mid 2 d} \frac{2 p^{\frac{3 n(n+1)}{2}}}{E_{p^{3}}(S)} \cdot \prod_{p \nmid 2 d} \frac{2 p^{\frac{n(n+1)}{2}}}{E_{p}(S)} \\
& =4 d^{\frac{n+2}{2}} B \cdot \frac{(2 \pi)^{\frac{n}{2}}}{(n-1) ! !} \prod_{p \mid 2 d} \frac{2 p^{\frac{3 n(n+1)}{2}}}{E_{p^{3}}(S)} \cdot \prod_{k=1}^{\frac{n}{2}} \prod_{p \mid 2 d}\left(1-p^{-2 k}\right) \\
& =4 d^{\frac{n+2}{2}} B \cdot \frac{(2 \pi)^{\frac{n}{2}}}{(n-1) ! !} \frac{\left(2^{\frac{n-1}{2}}+C\right)}{2^{\frac{n+3}{2}}} \prod_{p \mid d} \frac{\left(p^{\frac{n}{2}}+\left(\frac{-1}{p}\right)^{\frac{n}{2}}\right)}{2 p^{\frac{n+2}{2}}} \\
& =\frac{B}{2^{\frac{n}{2}+\omega(d)}}\left(2^{\frac{n}{2}}+2^{\frac{1}{2}} C\right) \prod_{p \mid d}\left(p^{\frac{n}{2}}+\left(\frac{-1}{p}\right)^{\frac{n}{2}}\right) \cdot \frac{(2 \pi)^{\frac{n}{2}}}{(n-1) ! !} .
\end{aligned}
$$

Corollary 4. If $n$ is odd, then the Euler characteristic of $\Gamma_{d}^{n}$ is zero. If $n$ is even, then the Euler characteristic of $\Gamma_{d}^{n}$ is given by

$$
\chi\left(\Gamma_{d}^{n}\right)=\frac{(-1)^{\frac{n}{2}} B}{2^{\frac{n}{2}+\omega(d)}}\left(2^{\frac{n}{2}}+2^{\frac{1}{2}} C\right) \prod_{p \mid d}\left(p^{\frac{n}{2}}+\left(\frac{-1}{p}\right)^{\frac{n}{2}}\right) .
$$

Proof. The group $\Gamma_{d}^{n}$ is finitely generated, and so $\Gamma_{d}^{n}$ has a torsion-free subgroup of finite index by Selberg's Lemma. If $n$ is odd, then $\chi\left(\Gamma_{d}^{n}\right)=0$, since odd dimensional hyperbolic manifolds of finite volume have zero Euler characteristic. If $n$ is even, then the Gauss-Bonnet Theorem implies that

$$
\operatorname{vol}\left(H^{n} / \Gamma_{d}^{n}\right)=(-1)^{\frac{n}{2}} \chi\left(\Gamma_{d}^{n}\right) \cdot \frac{(2 \pi)^{\frac{n}{2}}}{(n-1) ! !} .
$$

The result now follows immediately from Theorem 4 . 
Using the formulas in Theorem 4, we can easily compute the volume of $H^{n} / \Gamma_{d}^{n}$. For example, we have

$$
\begin{aligned}
\operatorname{vol}\left(H^{3} / \Gamma_{3}^{3}\right) & =\frac{5 \sqrt{3}}{64} L(2,-3), \\
\operatorname{vol}\left(H^{3} / \Gamma_{7}^{3}\right) & =\frac{7 \sqrt{7}}{64} L(2,-7), \\
\operatorname{vol}\left(H^{5} / \Gamma_{5}^{5}\right) & =\frac{15 \sqrt{5}}{2048} L(3,5), \\
\operatorname{vol}\left(H^{7} / \Gamma_{7}^{7}\right) & =\frac{49 \sqrt{7}}{98304} L(4,-7) .
\end{aligned}
$$

These four volumes were reported on pages 344 and 345 of [8], with some sign differences due to different definitions of $\Gamma_{d}^{n}$ and $L(s, D)$. In [8], the group $\Gamma_{d}^{n}$ was denoted by $\Gamma_{-d}^{n}$, and $L$-functions were defined via Legendre symbols rather than Kronecker symbols.

\section{The volumes of Mcleod's Polytopes}

J. Mcleod 10] has determined the reflection subgroup of $\Gamma_{3}^{n}$ and has shown that it has finite index if and only if $n \leq 13$. Let $P^{n}$ be the hyperbolic Coxeter fundamental domain of the reflection subgroup of $\Gamma_{3}^{n}$ determined by Mcleod in [10. Mcleod showed that the reflection subgroup of $\Gamma_{3}^{n}$ has index equal to the order of the symmetry group $\operatorname{Sym}\left(P^{n}\right)$ of $P^{n}$; moreover, Mcleod determined $\operatorname{Sym}(P)$ for $n \leq 13$. Hence, for $n \leq 13$, we have that

$$
\operatorname{vol}\left(P^{n}\right)=\left|\operatorname{Sym}\left(P^{n}\right)\right| \operatorname{vol}\left(H^{n} / \Gamma_{3}^{n}\right) .
$$

Table 1 lists the volumes of $H^{n} / \Gamma_{3}^{n}$ and $P^{n}$ for $n \leq 13$ computed using our formulas.

The polygon $P^{2}$ is $30^{\circ}-45^{\circ}$ right triangle, and so we can compute the area of $P^{2}$ by the classical angle defect formula

$$
\operatorname{vol}\left(P^{2}\right)=\pi-\left(\frac{\pi}{2}-\frac{\pi}{4}-\frac{\pi}{6}\right)=\frac{\pi}{12}
$$

The polyhedron $P^{3}$ is an orthotetrahedron with angles $\frac{\pi}{6}, \frac{\pi}{3}, \frac{\pi}{4}$. By Theorem 10.4.5 and the duplication formulas 10.4.9 and 10.4.10 in [13], we have that

$$
\operatorname{vol}\left(P^{3}\right)=\frac{5}{16} \pi\left(\frac{\pi}{3}\right)
$$

where $\pi(\theta)$ is the Lobachevsky function. By Formula 2 in Milnor [11, we have that

$$
\pi(\theta)=\frac{1}{2} \sum_{k=1}^{\infty} \sin (2 k \theta) k^{-2}
$$

Observe that

$$
\sin (2 k \pi / 3)=\frac{\sqrt{3}}{2}\left(\frac{-3}{k}\right) .
$$

Hence we have that

$$
\operatorname{vol}\left(P^{3}\right)=\frac{5 \sqrt{3}}{64} L(2,-3) .
$$




\begin{tabular}{rlcll}
$n$ & $\operatorname{vol}\left(\Gamma_{3}^{n}\right)$ & $\left|\operatorname{Sym}\left(P^{n}\right)\right|$ & $\operatorname{vol}\left(P^{n}\right)$ & $\left|\operatorname{vol}\left(P^{n}\right)\right|$ \\
\hline 2 & $\frac{\pi}{12}$ & 1 & $\frac{\pi}{12}$ & $2.617993878 * 10^{-1}$ \\
3 & $\frac{5 \sqrt{3}}{64} L(2,-3)$ & 1 & $\frac{5 \sqrt{3}}{64} L(2,-3)$ & $1.057230840 * 10^{-1}$ \\
4 & $\frac{\pi^{2}}{288}$ & 1 & $\frac{\pi^{2}}{288}$ & $3.426945973 * 10^{-2}$ \\
5 & $\frac{\sqrt{3}}{320} L(3,12)$ & 1 & $\frac{\sqrt{3}}{320} L(3,12)$ & $5.358748797 * 10^{-3}$ \\
6 & $\frac{13 \pi^{3}}{604800}$ & 1 & $\frac{13 \pi^{3}}{604800}$ & $6.664708943 * 10^{-4}$ \\
7 & $\frac{51 \sqrt{3}}{1146880} L(4,-3)$ & 1 & $\frac{51 \sqrt{3}}{1146880} L(4,-3)$ & $7.240232999 * 10^{-5}$ \\
8 & $\frac{697 \pi^{4}}{9144576000}$ & 1 & $\frac{697 \pi^{4}}{9144576000}$ & $7.424525364 * 10^{-6}$ \\
9 & $\frac{L(5,12)}{716800 \sqrt{3}}$ & 1 & $\frac{L(5,12)}{716800 \sqrt{3}}$ & $8.051559421 * 10^{-7}$ \\
10 & $\frac{341 \pi^{5}}{987614208000}$ & 2 & $\frac{341 \pi^{4}}{493807104000}$ & $2.113228256 * 10^{-7}$ \\
11 & $\frac{403 L(6,-3)}{12918456320 \sqrt{3}}$ & 2 & $\frac{403 L(6,-3)}{6459228160 \sqrt{3}}$ & $3.546550442 * 10^{-8}$ \\
12 & $\frac{50443 \pi^{6}}{12428137193472000}$ & 2 & $\frac{50443 \pi^{6}}{6214068596736000}$ & $7.804122909 * 10^{-9}$ \\
13 & $\frac{691 L(7,12)}{344408064000 \sqrt{3}}$ & 4 & $\frac{691 L(7,12)}{86102016000 \sqrt{3}}$ & $4.633381297 * 10^{-9}$
\end{tabular}

TABLE 1. The volumes of Mcleod's hyperbolic Coxeter polytopes

For even $n$, we can compute the volume of the polytope $P^{n}$ using the GaussBonnet Theorem which implies that

$$
\operatorname{vol}\left(P^{n}\right)=(-1)^{\frac{n}{2}} \chi\left(\mathrm{P}^{n}\right) \cdot \frac{(2 \pi)^{\frac{n}{2}}}{(n-1) ! !}
$$

where $\chi\left(\mathrm{P}^{n}\right)$ is the Euler characteristic of the Coxeter group $\mathrm{P}^{n}$ defined by $P^{n}$. By Proposition 3 of Chiswell [3], we have that

$$
\chi\left(\mathrm{P}^{n}\right)=\sum_{\Delta} \frac{(-1)^{|\Delta|}}{\left|C_{\Delta}\right|}
$$

where the sum is over all subgraphs $\Delta$ of the Coxeter graph of $\mathrm{P}^{n}$ such that the Coxeter group $C_{\Delta}$ defined by $\Delta$ is finite. Here $|\Delta|$ is the number of vertices of $\Delta$. From the Coxeter graph of $\mathrm{P}^{n}$ given in Mcleod [10, we computed the volumes of $P^{n}$ for all even $n \leq 12$ using Chiswell's Euler characteristic formula and the Gauss-Bonnet Theorem. The volumes agree with the volumes given in Table 1.

For odd $n$, we computed the volume of $P^{n}$ by numerical integration. We found very close agreement with the numerical values in Table 1 in dimensions $3,5,7,9$. We found close agreement in dimensions 11 and 13, but the accuracy deteriorated to a $1 \%$ discrepancy in dimension 11 and a $3 \%$ discrepancy in dimension 13, which is not bad considering we numerically integrated an $n$-fold integral for $n=11,13$. After the agreement of all these alternate calculations of the volume of $P^{n}$ for all $n \leq 13$, we are confident with the correctness of our volume formulas derived using Siegel's analytic theory of quadratic forms. 


\section{A COMMEnSURABiLity RATiO}

M. Belolipetsky and V. Emery [2] proved that for each odd dimension $n \geq 5$ there is a unique orientable, noncompact, arithmetic, hyperbolic $n$-orbifold $H^{n} / \Delta_{n}$ of smallest volume. If $n \equiv 3 \bmod 4$, they proved that the volume of $\Delta_{n}$ is given by

$$
\operatorname{vol}\left(H^{n} / \Delta_{n}\right)=\frac{3^{\frac{n}{2}}}{2^{\frac{n-1}{2}}} L\left(\frac{n+1}{2},-3\right) \prod_{k=1}^{\frac{n-1}{2}} \frac{(2 k-1) !}{(2 \pi)^{2 k}} \zeta(2 k) .
$$

In terms of Bernoulli numbers, the above formula can be rewritten as

$$
\operatorname{vol}\left(H^{n} / \Delta_{n}\right)=\frac{3^{\frac{n}{2}} B}{2^{n-1}} L\left(\frac{n+1}{2},-3\right) .
$$

In [6], V. Emery mentioned that $\Delta_{n}$ is commensurable to $\Gamma_{3}^{n}$ when $n \equiv 3 \bmod 4$. We now compute the ratio $\operatorname{vol}\left(H^{n} / \Gamma_{3}^{n}\right) / \operatorname{vol}\left(H^{n} / \Delta_{n}\right)$ for $n \equiv 3 \bmod 4$. By Theorem 4 , we have that

$$
\operatorname{vol}\left(H^{n} / \Gamma_{3}^{n}\right)=\frac{3^{\frac{n}{2}} B}{2^{n+1}}\left(2^{\frac{n-1}{2}}+(-1)^{\frac{n+1}{4}}\right)\left(2^{\frac{n+1}{2}}+1\right) L\left(\frac{n+1}{2},-3\right) .
$$

Therefore, we have

$$
\frac{\operatorname{vol}\left(H^{n} / \Gamma_{3}^{n}\right)}{\operatorname{vol}\left(H^{n} / \Delta_{3}\right)}=\frac{1}{4}\left(2^{\frac{n-1}{2}}+(-1)^{\frac{n+1}{4}}\right)\left(2^{\frac{n+1}{2}}+1\right)
$$

Although $\Delta_{n}$ is considered in [2] only for $n \geq 5$, we can define $\Delta_{3}$ to be $\operatorname{PGL}\left(2, \mathcal{O}_{3}\right)$ where $\mathcal{O}_{3}$ is the ring of integers of $\mathbb{Q}(\sqrt{-3})$. Then $\Delta_{3}$ is arithmetic and Meyerhoff [12] proved that $H^{3} / \Delta_{3}$ has minimum volume among all orientable, noncompact, hyperbolic 3 -orbifolds. The group $\Delta_{3}$ is the orientation preserving subgroup of a hyperbolic Coxeter group of type $[3,3,6]$. The above formula for $\operatorname{Vol}\left(H^{3} / \Delta_{3}\right)$ gives the correct volume for $H^{3} / \Delta_{3}$. Hence the above formula for $\operatorname{vol}\left(H^{3} / \Gamma_{3}^{3}\right) / \operatorname{vol}\left(H^{3} / \Delta_{3}\right)$ gives the correct value $5 / 4$.

The groups $\Gamma_{3}^{3}$ and $\Delta_{3}$ are commensurable. The relationship between $\Gamma_{3}^{3}$ and $\Delta_{3}$ is explained by the commensurability diagram on page 130 of Johnson et al. 9]. The group $\Gamma_{3}^{3}$ is a hyperbolic Coxeter group of type $[4,3,6]$. The Coxeter group $[4,3,6]$ has a Coxeter subgroup of type $\left[6,3^{1,1}\right]$ of index 2 . Hence the orientation preserving subgroup of $\left[6,3^{1,1}\right]$ has index 4 in $[4,3,6]$. Now the Coxeter tetrahedron of type $\left[6,3^{1,1}\right]$ can be subdivided into 5 copies of the Coxeter tetrahedron of type $[3,3,6]$. See Figure 1. Hence $\left[6,3^{1,1}\right]$ is conjugate to a subgroup of $[3,3,6]$ of index 5 . Therefore, the orientation preserving subgroup of $\left[6,3^{1,1}\right]$ is conjugate to a subgroup of $\Delta_{3}$ of index 5 . Thus, the ratio $5 / 4$ faithfully represents the commensurability relationship between $\Gamma_{3}^{3}$ and $\Delta_{3}$, that is, $\Gamma_{3}^{3}$ has a subgroup of index 4 that is conjugate to a subgroup of $\Delta_{3}$ of index 5 .

We now turn our attention to dimension 7 . Our volume ratio formula gives that

$$
\operatorname{vol}\left(H^{7} / \Gamma_{3}^{7}\right) / \operatorname{vol}\left(H^{7} / \Delta_{7}\right)=153 / 4 \text {. }
$$

By the main theorem of Hild [7], the group $\Delta_{7}$ is a subgroup of index two of a discrete group $\hat{\Delta}_{7}$ of isometries of $H^{7}$ such that $\Delta_{7}$ is the orientation preserving subgroup of $\hat{\Delta}_{7}$. The group $\hat{\Delta}_{7}$ is generated by a Coxeter subgroup $\bar{\Delta}_{7}$ of index 2 of type $\left[3^{3,2,2}\right]$ and an involution $\sigma$ that acts as the mirror symmetry of the Coxeter system defining $\bar{\Delta}_{7}$. The involution $\sigma$ is orientation preserving, since $\sigma$ transposes two pairs of Coxeter generators of $\bar{\Delta}_{7}$. Hence $\Delta_{7}$ is generated by the orientation preserving subgroup of $\bar{\Delta}_{7}$ and $\sigma$. 


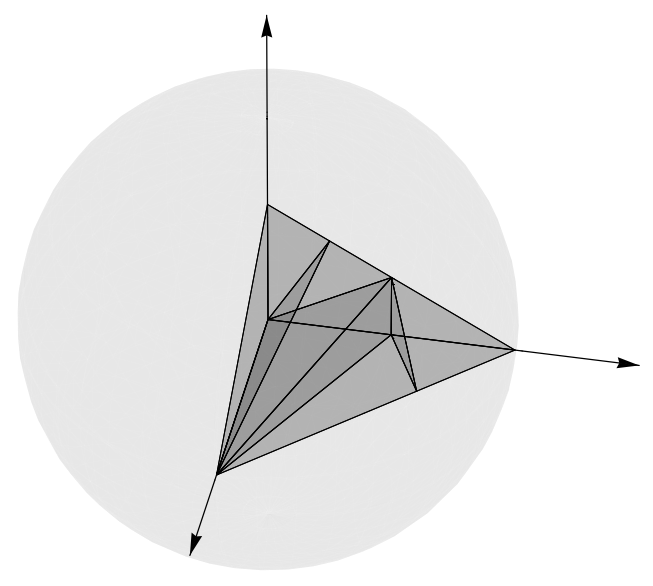

Figure 1. The subdivision of the Coxeter tetrahedron of type $\left[6,3^{1,1}\right]$ into 5 copies of the Coxeter tetrahedron of type $[3,3,6]$ shown in the projective ball model of hyperbolic 3 -space with the coordinate axes shown.

We explicitly defined the group $\bar{\Delta}_{7}$ to be the group generated by the reflections in the sides of the hyperbolic Coxeter 7 -simplex $\Delta$ whose sides have Lorentz normal unit vectors listed in the columns of the matrix

$$
\left(\begin{array}{rrrrrrrr}
0 & 0 & 0 & 0 & 0 & -\frac{1}{2} & 0 & 1 \\
0 & -\frac{1}{2} & 0 & 0 & 0 & \frac{1}{2} & 0 & \frac{1}{2} \\
0 & \frac{1}{2} & 0 & -\frac{1}{2} & 0 & \frac{1}{2} & 0 & \frac{1}{2} \\
0 & 0 & 0 & \frac{1}{2} & 0 & 0 & -1 & \frac{1}{2} \\
0 & 0 & 0 & \frac{1}{2} & -1 & \frac{1}{2} & 0 & 0 \\
0 & \frac{1}{2} & -1 & \frac{1}{2} & 0 & 0 & 0 & 0 \\
-1 & \frac{1}{2} & 0 & 0 & 0 & 0 & 0 & 0 \\
0 & 0 & 0 & 0 & 0 & 0 & 0 & \frac{\sqrt{3}}{2}
\end{array}\right) .
$$

The involution $\sigma$ acts as a symmetry of $\Delta$. The Lorentzian matrix of $\sigma$ is

$$
\left(\begin{array}{rrrrrrrr}
-2 & 0 & 0 & 0 & 0 & 0 & 0 & \sqrt{3} \\
0 & 1 & 0 & 0 & 0 & 0 & 0 & 0 \\
0 & 0 & 1 & 0 & 0 & 0 & 0 & 0 \\
0 & 0 & 0 & 0 & 1 & 0 & 0 & 0 \\
0 & 0 & 0 & 1 & 0 & 0 & 0 & 0 \\
0 & 0 & 0 & 0 & 0 & 1 & 0 & 0 \\
0 & 0 & 0 & 0 & 0 & 0 & 1 & 0 \\
-\sqrt{3} & 0 & 0 & 0 & 0 & 0 & 0 & 2
\end{array}\right) .
$$

In 1996, we discovered that $\Gamma_{3}^{7}$ is a Coxeter reflection group defined by the Coxeter graph in Figure 1 of [10, for $n=7$, using Vinberg's algorithm [17. We explicitly defined the group $\Gamma_{3}^{7}$ to be the group generated by the reflections in the sides of the hyperbolic Coxeter 7-dimensional polytope $P^{7}$ whose sides have Lorentz 
normal unit vectors listed in the columns of the matrix

$$
\left(\begin{array}{rrrrrrrrr}
-\frac{1}{\sqrt{2}} & 0 & 0 & 0 & 0 & 0 & 0 & \sqrt{\frac{3}{2}} & \frac{1}{\sqrt{2}} \\
\frac{1}{\sqrt{2}} & -\frac{1}{\sqrt{2}} & 0 & 0 & 0 & 0 & 0 & 0 & \frac{1}{\sqrt{2}} \\
0 & \frac{1}{\sqrt{2}} & -\frac{1}{\sqrt{2}} & 0 & 0 & 0 & 0 & 0 & \frac{1}{\sqrt{2}} \\
0 & 0 & \frac{1}{\sqrt{2}} & -\frac{1}{\sqrt{2}} & 0 & 0 & 0 & 0 & \frac{1}{\sqrt{2}} \\
0 & 0 & 0 & \frac{1}{\sqrt{2}} & -\frac{1}{\sqrt{2}} & 0 & 0 & 0 & \frac{1}{\sqrt{2}} \\
0 & 0 & 0 & 0 & \frac{1}{\sqrt{2}} & -\frac{1}{\sqrt{2}} & 0 & 0 & 0 \\
0 & 0 & 0 & 0 & 0 & \frac{1}{\sqrt{2}} & -1 & 0 & 0 \\
0 & 0 & 0 & 0 & 0 & 0 & 0 & \frac{1}{\sqrt{2}} & \sqrt{\frac{3}{2}}
\end{array}\right) .
$$

In 1996, we discovered by a coset enumeration program that $\bar{\Delta}_{7} \cap \Gamma_{3}^{7}$ has index 2295 in $\bar{\Delta}_{7}$ and index 60 in $\Gamma_{3}^{7}$. The involution $\sigma$ is an element of $\Gamma_{3}^{7}$ and so $\Delta_{7} \cap \Gamma_{3}^{7}$ has index 2295 in $\Delta_{7}$ and index 60 in $\Gamma_{3}^{7}$. The commensurability ratio is $2295 / 60=153 / 4$. We were disappointed that we could not find representations of $\Delta_{7}$ and $\Gamma_{3}^{7}$ such that $\Delta_{7} \cap \Gamma_{3}^{7}$ has index 153 in $\Delta_{7}$ and index 4 in $\Gamma_{3}^{7}$, but we suspect that the representations that we found give the smallest possible indices.

In 1996, we found a discrete group $\Gamma$ of isometries of $H^{7}$ that corresponds to the group of positive units of the quadratic form defined by the diagonal matrix $\operatorname{diag}(1,1,1,1,1,1,3,-1)$ such that $\bar{\Delta}_{7} \cap \Gamma$ has index 119 in $\bar{\Delta}_{7}$ and index 4 in $\Gamma$. Moreover $\operatorname{vol}\left(H^{7} / \Gamma\right) / \operatorname{vol}\left(H^{7} / \bar{\Delta}_{7}\right)=119 / 4$, and so the ratio $119 / 4$ faithfully represents the commensurability relationship between $\bar{\Delta}_{7}$ and $\Gamma$. This computation was reported on page 345 of 8 . The story of the computation of the volume of $H^{7} / \Gamma$ will have to wait for another day.

\section{REFERENCES}

[1] T. M. Apostol, Introduction to Analytic Number Theory, Undergraduate Texts in Math., Springer-Verlag, New York, 1976.

[2] M. Belolipetsky and V. Emery, On volumes of arithmetic quotients of $\mathrm{PO}(n, 1)^{\circ}, n$ odd, preprint, 2010.

[3] I. M. Chiswell, The Euler characteristic of graph products and of Coxeter groups, In: Discrete Groups and Geometry, London Math. Soc. Lec. Notes Ser. 173 (1992), 36-46.

[4] H. Cohen, A Course in Computational Algebraic Number Theory, Graduate Texts in Math., vol. 138, Springer-Verlag, Berlin, Heidelberg, and New York, 1993.

[5] L. E. Dickson, Linear Groups with an Exposition of the Galois Field Theory, B. G. Teubner, Leipzig, 1901.

[6] V. Emery, Even unimodular Lorentzian lattices and hyperbolic volume, preprint 2011.

[7] T. Hild, The cusped hyperbolic orbifolds of minimal volume in dimensions less than ten, J. Algebra 313 (2007), 208-222.

[8] N. W. Johnson, R. Kellerhals, J. G. Ratcliffe, and S. T. Tschantz, The size of a hyperbolic Coxeter simplex, Transform. Groups. 4 (1999), 329-353.

[9] N. W. Johnson, R. Kellerhals, J. G. Ratcliffe, and S. T. Tschantz, Commensurability classes of hyperbolic Coxeter groups, Linear Algebra Appl. 345 (2002), 119-147.

[10] J. Mcleod, Hyperbolic reflection groups associated to the quadratic forms $-3 x_{0}^{2}+x_{1}^{2}+\cdots+x_{n}^{2}$, Geom. Dedicata 152 (2011), 1-16.

[11] J. Milnor, Hyperbolic geometry: the first 150 years, Bull. Amer. Math. Soc. 6 (1982), 9 - 24.

[12] R. Meyerhoff, The cusped hyperbolic 3-orbifold of minimum volume, Bull. Amer. Math. Soc. 13 (1985), 154-156.

[13] J.G. Ratcliffe, Foundations of Hyperbolic Manifolds, Second Edition, Graduate Texts in Math., vol. 149, Springer-Verlag, Berlin, Heidelberg, and New York, 2006. 
ON VOLUMES OF HYPERBOLIC COXETER POLYTOPES AND QUADRATIC FORMS 17

[14] J. G. Ratcliffe and S. T. Tschantz, Volumes of integral congruence hyperbolic manifolds $J$. Reine Angew. Math. 488 (1997), 55-78.

[15] C. L. Siegel, Über die analytische Theorie der quadratischen Formen, Ann. Math. 36 (1935), 527-606.

[16] C. L. Siegel, Über die analytische Theorie der quadratischen Formen II, Ann. Math. 37 (1936), 230-263.

[17] È. B. Vinberg, On groups of unit elements of certain quadratic forms, Math. Sbornik 16, (1972), 17-35.

Department of Mathematics, Vanderbilt University, Nashville, TN 37240

E-mail address: j.g.ratcliffe@vanderbilt.edu 